\title{
The Semantics of Program Slicing and Program Integration
}

\author{
Thomas Reps and Wuu Yang \\ Computer Sciences Department \\ University of Wisconsin - Madison \\ 1210 W. Dayton Street \\ Madison, WI 53706 USA
}

\begin{abstract}
A slice of a program with respect to a program point $p$ and variable $x$ consists of all statements of the program that might affect the value of $x$ at point $p$. Slices can be extracted particularly easily from a program representation called a program dependence graph, originally introduced as an intermediate program representation for performing optimizing, vectorizing, and parallelizing transformations. Such slices are of a slightly restricted form: rather than permitting a program to be sliced with respect to program point $p$ and an arbitrary variable, a slice must be taken with respect to a variable that is defined at or used at $p$.
\end{abstract}

This paper concerns the relationship between the execution behavior of a program and the execution behavior of its slices. Our main results about slicing are those stated as the Slicing Theorem and the Termination Theorem. The Slicing Theorem demonstrates that a slice captures a portion of a program's behavior in the sense that, for any initial state on which the program halts, the program and the slice compute the same sequence of values for each element of the slice. The Termination Theorem demonstrates that if a program is decomposed into (two or more) slices, the program halts on any state for which all the slices halt.

These results are then used to provide semantic justification for a program-integration algorithm of Horwitz, Prins, and Reps.

\section{INTRODUCTION}

The slice of a program with respect to program point $p$ and variable $x$ consists of all statements and predicates of the program that might affect the value of $x$ at point $p$ [11]. Program slicing can be used to isolate individual computation threads within a program, which can help a programmer understand complicated code. Program slicing is also used by the algorithm for automatically integrating program variants described in [2] and [7]; slices are used to compute a safe approximation to the computation threads that have changed between a program $P$ and a modified version of $P$, and to help determine whether two different modifications to $P$ interfere.

This work was supported in part by a David and Lucile Packard Fellowship for Science and Engineering, by the National Science Foundation under grant DCR-8552602, by the Defense Advanced Research Projects Agency, monitored by the Office of Naval Research under contract N00014-88-K-0590, as well as by grants from IBM, DEC, and Xerox. 
The original algorithm given for program slicing was expressed as a sequence of data flow analysis problems [11]. An alternative (and more practical) approach was put forward in [5], where it was pointed out that the slice of a program with respect to an initial set of vertices could be computed by walking backwards over the edges of the program dependence graphs being proposed as the internal representation of programs in a program development environment. The kind of slicing that can be performed using a program dependence graph is, however, somewhat restricted: rather than permitting a program to be sliced with respect to program point $p$ and an arbitrary variable, a slice must be taken with respect to a variable that is defined at or $u s e d$ at $p$. It is this restricted kind of slice that is studied here.

This paper presents results that characterize the relationship between the execution behavior of a program and the execution behavior of its slices. Our main results on slicing are those stated as the Slicing Theorem and the Termination Theorem. The Slicing Theorem demonstrates that a slice captures a portion of a program's behavior in the sense that, for any initial state on which the program halts, the program and the slice compute the same sequence of values for each element of the slice. The Termination Theorem demonstrates that if a program is decomposed into (two or more) slices, the program halts on any state for which all the slices halt.

These theorems are then used to provide semantic justification for the program-integration algorithm presented in $[2,7]$; the integration algorithm presented there either merges two program versions with a base version of the program or determines that the variants incorporate interfering changes. In the algorithm, slicing is used to determine which elements from the base program and its variants should be incorporated in the integrated program. The integrated program is created by (1) finding slices that represent the changed computation threads of the variant programs as well as the computation threads of the base program that are preserved in both variants, (2) combining these slices to form the merged graph, and (3) testing for interference by checking whether the slices that were combined to form the merged graph are preserved (as slices of the merged graph). (A prototype implementation of an integration tool based on this algorithm has been embedded in a program editor created using the Synthesizer Generator $[6,8]$.

The Slicing and Termination Theorems are used to prove a theorem, the Integration Theorem, that characterizes the execution behavior of the integrated program in terms of the behaviors of the base program and the two variants; the Integration Theorem demonstrates that the integrated program produced by a successful integration preserves the changed behaviors of both variants as well as the behavior of the base program that is unchanged in both variants.

The remainder of the paper is organized as follows. Section 2 defines program dependence graphs and the operation of program slicing. Section 3 concerns the Feasibility Lemma, which states that a slice extracted from a program dependence graph corresponds to some program. Section 4 discusses our results on the semantics of slicing, the Slicing and Termination Theorems. In Section 5, we review the steps of the program-integration algorithm and then prove the Integration Theorem. Section 6 discusses the relation of the work described to previous work. 
All proofs except those pertaining directly to the Integration Theorem have been omitted, as they are somewhat lengthy structural inductions; full proofs can be found in [9].

\section{TERMINOLOGY AND NOTATION}

We are concerned with a restricted programming language with the following characteristics: expressions contain only scalar variables and constants; statements are either assignment statements, conditional statements, while loops, or a restricted kind of "output statement" called an end statement, which can only appear at the end of a program. An end statement names one or more of the variables used in the program. Thus a program is of the form:

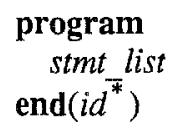

Our discussion of the language's semantics is in terms of the following informal model of execution. We assume a standard operational semantics for sequential execution of the corresponding flowchart (control flow graph): at any moment there is a single locus of control together with a global execution state mapping program variables to values; the execution of each assignment statement or predicate passes control to a single successor; the execution of each assignment statement changes the global execution state. An execution of the program on some initial state also yields a (possibly infinite) sequence of values for each predicate and assignment statement in the program; the $i^{t h}$ element in the sequence for program element $e$ consists of the value computed when $e$ is executed for the $i^{t h}$ time. The variables named in the end statement are those whose final values are of interest to the programmer; when execution terminates, the final state is defined on only those variables in the end statement.

\subsection{The Program Dependence Graph}

Different definitions of program dependence representations have been given, depending on the intended application; they are all variations on a theme introduced in [4], and share the common feature of having an explicit representation of data dependences (see below). The "program dependence graphs" defined in [1] introduced the additional feature of an explicit representation for control dependences (see below). The definition of program dependence graph given below differs from [1] in two ways. First, our definition covers only the restricted language described earlier, and hence is less general than the one given in [1]. Second, because of the particular needs of the program-integration problem, we omit certain classes of data dependence edges and introduce one new class. Despite these differences, the structures we define and those defined in [1] share the feature of explicitly representing both control and data dependences; therefore, we refer to our graphs as "program dependence graphs," borrowing the term from [1].

The program dependence graph (or PDG) for a program $P$, denoted by $G_{P}$, is a directed 
graph whose vertices are connected by several kinds of edges. ${ }^{1}$ The assignment statements and control predicates that occur in $P$ are represented by vertices of $G_{P}$. In addition, there are three other kinds of vertices: there is a distinguished vertex called the entry vertex; for each variable $x$ for which there is a path in $P$ 's control-flow graph on which $x$ is used before being defined, there is a vertex called the initial definition of $x$; and for each variable $x$ named in $P$ 's end statement, there is a vertex called the final use of $x$. There are two kinds of edges: control dependence edges and data dependence edges. The source of a control dependence edge is either the entry vertex or a predicate vertex and each edge is labeled either true or false. A control dependence edge from vertex $v_{1}$ to vertex $v_{2}$ means (roughly) that during execution, whenever the predicate represented by $v_{1}$ is evaluated and its value matches the label on the edge to $v_{2}$, then the program component represented by $v_{2}$ will eventually be executed.

A data dependence edge from vertex $v_{1}$ to vertex $v_{2}$ means that the program's behavior might change if the relative order of the components represented by $v_{1}$ and $v_{2}$ were reversed. There are two kinds of data dependence edges: flow edges and def-order edges. A flow edge runs from a vertex $v_{1}$ that represents an assignment to a variable $x$ to a vertex $v_{2}$ that represents a use of $x$ reached by assignment $v_{1}$. A flow dependence is loop carried if $v_{1}$ reaches $v_{2}$ along a path in the control-flow graph that includes the back-edge of a loop, and the loop encloses both $v_{1}$ and $v_{2}$; otherwise it is loop independent. A def-order edge runs from $v_{1}$ to $v_{2}$ when both vertices represent assignments to $x$, both $v_{1}$ and $v_{2}$ reach a common use of $x$, and $v_{1}$ lexically precedes $v_{2}{ }^{2}$

Example. Figure 1 shows an example program and its program dependence graph. The boldface arrows represent control dependence edges; dashed arrows represent def-order dependence edges; solid arrows represent loop-independent flow dependence edges; solid arrows with a hash mark represent loop-carried flow dependence edges.

\subsection{Program Slices}

For a vertex $s$ of a PDG $G$, the slice of $G$ with respect to $s$, written as $G / s$, is a graph containing all vertices on which $s$ has a transitive flow or control dependence (i.e. all vertices that can reach $s$ via flow or control edges): $V(G / s)=\left\{w \in V(G) \mid w \rightarrow{ }_{c, f} s\right\}$. We extend the definition to a set of vertices $S=\bigcup_{i} s_{i}$ as follows: $V(G / S)=V\left(G /\left(\bigcup_{i} s_{i}\right)\right)=\bigcup_{i} V\left(G / s_{i}\right)$.

\footnotetext{
${ }^{1}$ A directed graph $G$ consists of a set of vertices $V(G)$ and a set of edges $E(G)$, where $E(G) \subseteq V(G) \times V(G)$. Each edge $(b, c) \in E(G)$ is directed from $b$ to $c$; we say that $b$ is the source and $c$ the target of the edge.

${ }^{2}$ A flow dependence (respectively, control dependence) that exists from vertex $v_{1}$ to vertex $v_{2}$ will be denoted by $v_{1} \rightarrow_{f} v_{2}\left(v_{1} \rightarrow_{c} v_{2}\right)$. A def-order dependence from $v_{1}$ to $v_{2}$ where the definitions at both $v_{1}$ and $v_{2}$ reach $v_{3}$ will be denoted by $v_{1} \rightarrow{ }_{d o\left(v_{3}\right)} v_{2}$.
} 


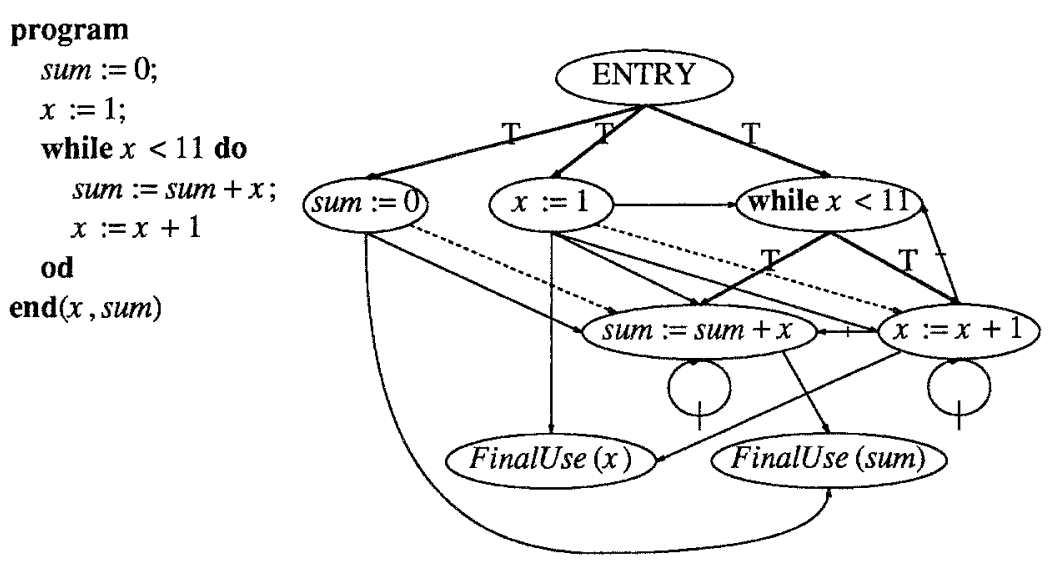

Figure 1. An example program, which sums the integers from 1 to 10 and leaves the result in the variable sum, and its program dependence graph. The boldface arrows represent control dependence edges, dashed arrows represent def-order dependence edges, solid arrows represent loop-independent flow dependence edges, and solid arrows with a hash mark represent loop-carried flow dependence edges.

The edges in the graph $G / S$ are essentially those in the subgraph of $G$ induced by $V(G / S)$, with the exception that a def-order edge $v \rightarrow_{d o(u)} w$ is only included if, in addition to $v$ and $w$, $V(G / S)$ also contains the vertex $u$ that is directly flow dependent on the definitions at $v$ and $w$. In terms of the three types of edges in a PDG we have:

$$
\begin{aligned}
E(G / S)= & \left\{\left(v \rightarrow_{f} w\right) \in E(G) \mid v, w \in V(G / S)\right\} \\
& \cup\left\{\left(v \rightarrow_{c} w\right) \in E(G) \mid v, w \in V(G / S)\right\} \\
& \cup\left\{\left(v \rightarrow_{d o(u)} w\right) \in E(G) \mid u, v, w \in V(G / S)\right\}
\end{aligned}
$$

Example. Figure 2 shows the graph resulting from taking a slice of the program dependence graph from Figure 1 with respect to the final-use vertex for $x$.

The following lemma captures a useful property of program slicing.

LEMMA. (Decomposition Lemma). For any collection $\cup_{i} s_{i}$ of program points, we have $\bigcup_{i}\left(G / s_{i}\right)=G / \cup_{i} s_{i}$

\section{THE FEASIBHLTY LEMMA}

Our first result concerns a syntactic property of slices: we say that a graph $G$ is a feasible program dependence graph iff $G$ is isomorphic to the program dependence graph of some program. We can show that for any program $P$ and vertex set $S$, the slice $G_{P} / S$ is a feasible PDG. 

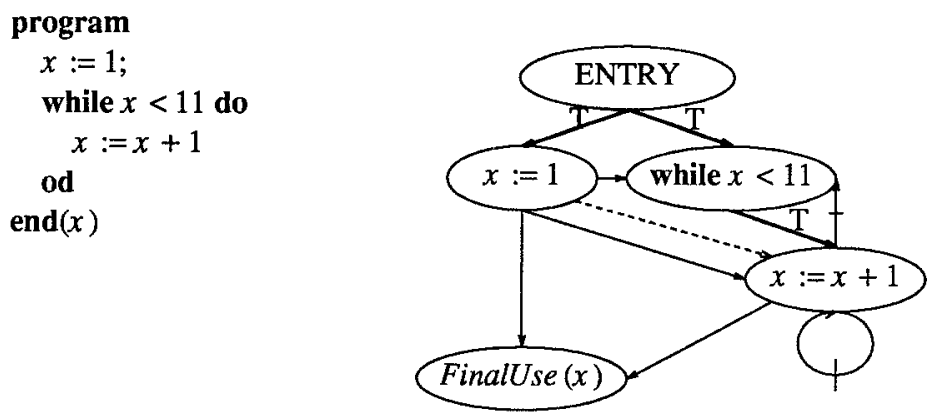

Figure 2. The graph that result from slicing the example from Figure 1 with respect to the final-use vertex for $x$, together with the one program to which it corresponds.

LEMMA. (FEASIBILITY LEMMA). For any program $P$, if $G_{Q}$ is a slice of $G_{P}$ (with respect to some set of vertices), then $G_{Q}$ is a feasible $P D G$.

The proof proceeds by showing that $G_{P} / S$ corresponds to the program whose elements are the statements and predicates in $V\left(G_{P} / S\right)$ arranged in the same order that they occur in $P$. (Note that there can be other programs whose program dependence graphs are isomorphic to $\left.G_{Q}.\right)$

Since a slice of a feasible program dependence graph is feasible, we can speak of "a slice of a program" as well as "a slice of a program dependence graph." We say program $Q$ is a slice of program $P$ with respect to a set of program points, $S$, when $G_{Q}$ is isomorphic to $G_{P} / S$, and write this as $P / S$.

\section{THE SEMANTICS OF PROGRAM SLICING}

We now turn to the relationship between the execution behaviors of a program and a slice of the program. Because of the way a program slice is derived from a program, it is not unreasonable to expect that the program and the slice exhibit similar execution behavior. However, because a diverging computation may be "sliced out," a program and a slice of the program do not necessarily exhibit identical execution behaviors; in particular, a slice may produce a result on some initial states for which the original program diverges. For example, the program shown below on the left always diverges, whereas the program on the right, obtained by slicing the left-handside program with respect to variable $x$ at the program's end statement, always converges: 


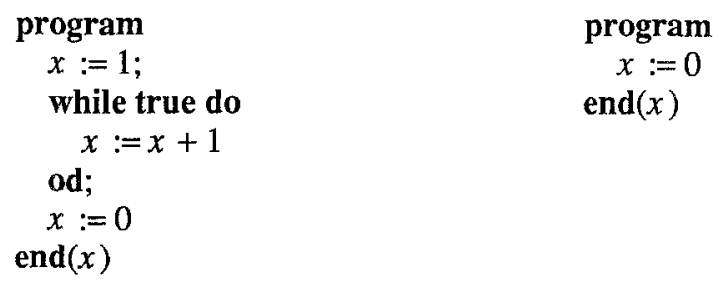

\subsection{The Slicing Theorem}

Our first result on the semantics of slicing is the following theorem, which asserts that a slice captures a portion of the program's behavior in the sense that, for any initial state on which the program halts, for each program point of the slice, the point in the slice and its corresponding point in the program compute the same sequence of values. ${ }^{3}$

THEOREM. (SLICING THEOREM). Let $Q$ be a slice of program $P$ with respect to a set of vertices. If $\sigma$ is a state on which $P$ halts, then for any state $\sigma^{\prime}$ that agrees with $\sigma$ on all variables for which there are initial-definition vertices in $G_{Q}:(1) Q$ halts on $\sigma^{\prime}$ (2) $P$ and $Q$ compute the same sequence of values at each program point of $Q$, and (3) the final states agree on all variables for which there are final-use vertices in $G_{Q}$.

The third clause of the theorem's conclusion is implied by the second clause; it is stated explicitly to emphasize what the theorem says about programs viewed as state-transformers.

The Slicing Theorem is proven by structural induction on $P$ 's abstract-syntax tree. In making this argument, it is necessary to discuss the state-transforming properties of a subtree. These can be characterized in terms of the subtree's imported and exported variables, defined as follows:

DEFINITION. The outgoing flow edges of a subtree $T$ consist of all the loop-independent flow edges whose source is in $T$ but whose target is not in $T$, together with all the loop-carried flow edges for which the source is in $T$ and the edge is carried by a loop that encloses $T$. Note that the target of an outgoing loop-carried flow edge may or may not be in $T$. The variables exported from a subtree $T$ are those defined at the source of an outgoing flow edge.

DEFINITION. The incoming flow edges of a subtree $T$ consist of all the loop-independent flow edges whose target is in $T$ but whose source is not in $T$, together with all the loop-carried flow edges for which the target is in $T$ and the edge is carried by a loop that encloses $T$. Note that

\footnotetext{
${ }^{3}$ In our case a program point may be (1) an assignment statement, (2) a control predicate, or (3) a final use of a variable in an end statement. By "computing the same sequence of values" at corresponding points we mean: (1) for an assignment statement the same sequence of values are assigned to the target variable; (2) for a predicate the same sequence of boolean values are produced; and (3) for a final use the same value for the variable is produced.
} 
the source of an incoming loop-carried flow edge may or may not be in $T$. The incoming deforder edges of a subtree $T$ consist of all the def-order edges whose target is in $T$ but whose source is not in $T$. The variables imported by a subtree $T$ are those defined at the source of an incoming flow edge or at the source of an incoming def-order edge.

Note that there are loop-independent flow edges to all final-use vertices of a program dependence graph; thus, the exported variables of a program $P$ consist of all the variables that occur in $P$ 's end statement. Similarly, there are loop-independent flow edges from all of the initialdefinition vertices; thus, the imported variables of a program $P$ consist of those variables that may get their values from the initial state.

There is a natural correspondence between subtrees in $P$ and subtrees in slices of $P$, defined as follows:

DEFINITION. Let $Q$ be a slice of $P$ with respect to some set of program points. For each subtree $U$ of $Q$ with root $u, U$ corresponds to the subtree of $P$ whose root is $u$. For each subtree $T$ of $P$, if the root $t$ of $T$ occurs in $Q, T$ corresponds to the subtree of $Q$ rooted at $t$; if $t$ does not occur in $Q, T$ corresponds to the empty tree.

Thus, for each subtree of $Q$, there is always a corresponding subtree of $P$, and vice versa, although for a subtree of $P$ the corresponding subtree of $Q$ may be the empty tree. (Note that the "corresponds to" relation respects the hierarchical structure of programs: children of roots of corresponding subtrees are the roots of corresponding subtrees.)

The Slicing Theorem is a consequence of a more general result, the Subtree Slicing Lemma, which characterizes the relationship between a subtree and a slice of the subtree in terms of the slice's imported and exported variables. The Lemma asserts that, for certain initial states, corresponding subtrees of a program and a slice of the program compute the same sequence of values at common program points.

LEMMA. (SUBTREE SLICING LEMMA). Let $Q$ be a slice of program $P$ with respect to a set of vertices. Let $T$ be a subtree of program $P$ and $U$ be the corresponding subtree of $Q$. If $\sigma$ is a state on which $T$ halts, then ( 1 ) $U$ halts on $\sigma$ ' where $\sigma$ and $\sigma$ ' agree on $U$ 's imported variables (as defined in the context given by $Q$ ), (2) $T$ and $U$ compute the same sequence of values at each program point of $U$, and (3) the final states agree on $U$ 's exported variables (as defined in the context given by $Q$ ).

The Slicing Theorem is simply the Subtree Slicing Lemma specialized to the case when subtree $T$ is the entire program $P$.

\subsection{The Termination Theorem}

The Slicing Theorem tells us that if a program terminates on some initial state then (on sufficiently similar initial states) the program's slices also terminate. The Termination Theorem looks at this relationship from the opposite point of view; it tells us that if a program is decomposed into two slices, the termination of the slices on some state implies that the program ter- 
minates on that state as well.

THEOREM. (TERMINATION THEOREM). Let $P$ be a program. Suppose $X$ and $Y$ are sets of vertices such that $G_{P}=\left(G_{P} / X\right) \cup\left(G_{P} / Y\right)$. If $P / X$ and $P / Y$ halt on a state $\sigma$, then $P$ halts on $\sigma$ as well.

Note that the Termination Theorem and clause (1) of the Slicing Theorem are complementary: clause (1) of the Slicing Theorem asserts that if a program terminates then each slice also terminates; the Termination Theorem asserts that when a program can be decomposed into two slices, if each slice terminates then the program terminates. We can then apply clause (2) of the Slicing Theorem to conclude that the two slices (collectively) compute the same sequence of values as the entire program.

It is straightforward to generalize the Termination Theorem to the case where the program is decomposed into more than two slices. The case when the program is decomposed into three slices is used in the proof of the Integration Theorem, which is given in the next section. (The integrated program that is the subject of the proof is formed by taking the union of three slices.)

COROLlaRY. Let $P$ be a program. Suppose $X, Y$, and $Z$ are sets of vertices such that $G_{P}=\left(G_{P} / X\right) \cup\left(G_{P} / Y\right) \cup\left(G_{P} / Z\right)$. If $P / X, P / Y$, and $P / Z$ halt on a state $\sigma$, then $P$ halts on $\sigma$ as well.

\section{THE SEMANTICS OF PROGRAM INTEGRATION}

An algorithm for integrating several related, but different variants of a base program (or determining whether the variants incorporate interfering changes) has been presented in [2,7]. The algorithm presented there, called Integrate, takes as input three programs $A, B$, and Base, where $A$ and $B$ are two variants of $B$ ase. As we show below, whenever the changes made to $B$ ase to create $A$ and $B$ do not "interfere" (in the sense defined below), Integrate produces a program $M$ that exhibits the changed execution behavior of $A$ and $B$ with respect to $B$ ase as well as the execution behavior preserved in all three versions.

The first step of Integrate determines slices that represent a safe approximation to the changed computation threads of $A$ and $B$ and the computation threads of Base preserved in both $A$ and $B$; the second step combines these slices to form the merged graph $G_{M}$; the third step tests $G_{M}$ for interference.

\section{Step 1: Determining changed and preserved computation threads}

If the slice of variant $G_{A}$ at vertex $v$ differs from the slice of $G_{\text {Base }}$ at $v$, then $G_{A}$ and $G_{B a s e}$ may compute different values at $v$. In other words, vertex $v$ is a site that potentially exhibits changed behavior in the two programs. Thus, we define the affected points of $G_{A}$ with respect to $G_{B a s e}$, denoted by $A P_{A, B a s e}$, to be the subset of vertices of $G_{A}$ whose slices in $G_{B a s e}$ and $G_{A}$ differ $A P_{A, B a s e}=\left\{v \in V\left(G_{A}\right) \mid G_{B a s e} / v \neq G_{A} / v\right\}$. We define $A P_{B, \text { Base }}$ similarly. It follows that the slices $G_{A} / A P_{A, B a s e}$ and $G_{B} / A P_{B, B a s e}$ capture the respective computation threads of $A$ 
and $B$ that differ from Base.

The preserved computation threads of Base in $A$ correspond to the slice $G_{B a s e} / \overline{A P}_{A, \text { Base }}$, where $\overline{A P}_{A, \text { Base }}$ is the complement of $A P_{A, \text { Base }}: \overline{A P}_{A, \text { Base }}=V\left(G_{A}\right)-A P_{A, \text { Base }}$. We define $\overline{A P}_{B, B \text { ase }}$ similarly. Thus, the unchanged computation threads common to both $A$ and $B$ are captured by the following slice: $G_{\text {Base }} /\left(\overline{A P}_{A, \text { Base }} \cap \overline{A P}_{B, \text { Base }}\right)$.

\section{Step 2: Forming the merged graph}

The merged program dependence graph, $G_{M}$, is formed by unioning the three slices that represent the changed and preserved computation threads of the two variants:

$G_{M}=\left(G_{A} / A P_{A, \text { Base }}\right) \cup\left(G_{B} / A P_{B, \text { Base }}\right) \cup\left(G_{\text {Base }} /\left(\overline{A P}_{A, \text { Base }} \cap \overline{A P}_{B, \text { Base }}\right)\right)$.

\section{Step 3: Testing for interference}

There are two possible ways by which the graph $G_{M}$ may fail to represent a satisfactory integrated program; both types of failure are referred to as "interference." The first interference criterion is based on a comparison of slices of $G_{A}, G_{B}$, and $G_{M}$. The slices $G_{A} / A P_{A, B a s e}$ and $G_{B} / A P_{B, B a s e}$ represent the changed computation threads of programs $A$ and $B$ with respect to Base. $A$ and $B$ interfere if $G_{M}$ does not preserve these slices; that is, there is interference of this kind if either $G_{M} / A P_{A, B a s e} \neq G_{A} / A P_{A, \text { Base }}$ or $G_{M} / A P_{B, \text { Base }} \neq G_{B} / A P_{B, \text { Base }}$.

The final step of the integration method involves reconstituting a program from the merged program dependence graph. However, it is possible that there is no such program; that is, the merged graph may be an infeasible program dependence graph. This is the second kind of interference that may occur. (The reader is referred to [2] for a discussion of reconstructing a program from the merged program dependence graph and the inherent difficulties of this problem.) If neither kind of interference occurs, one of the programs that corresponds to the graph $G_{M}$ is returned as the result of the integration operation.

\section{The Integration Theorem}

The Slicing and Termination Theorems, together with the definition of the merged graph $G_{M}$, can be used to prove a theorem that characterizes the execution behavior of the integrated program in terms of the behaviors of the base program and the two variants. Stated informally, $M$ preserves the changed behaviors of both $A$ and $B$ (with respect to Base) as well as the unchanged behavior of all three.

The merged program dependence graph, $G_{M}$, is formed by unioning the three slices $G_{A} / A P_{A, B a s e}, G_{B} / A P_{B, B a s e}$, and $G_{B a s e} /\left(\overline{A P}_{A, B a s e} \cap \overline{A P}_{B, \text { Base }}\right)$. Because the premise of the theorem is that integration succeeds, we know that $G_{M} / A P_{A, B a s e}=G_{A} / A P_{A, B a s e}$ and $G_{M} / A P_{B, \text { Base }}=G_{B} / A P_{B, \text { Base }}$. One detail that must be shown is that, in testing $G_{M}$ for interference, it is unnecessary to test whether

$G_{\text {Base }} /\left(\overline{A P}_{A, \text { Base }} \cap \overline{A P}_{B, \text { Base }}\right)=G_{M} /\left(\overline{A P}_{A, \text { Base }} \cap \overline{A P}_{B, \text { Base }}\right)$. 
This matter is addressed by the Preserved Behavior Lemma, stated and proven below, which shows that, regardless of whether or not the integration algorithm detects interference, the slice $G_{\text {Base }} /\left(\overline{A P}_{A, \text { Base }} \cap \overline{A P}_{B, \text { Base }}\right)$ is always preserved in $G_{M}$.

LEMMA. If $w \in A P_{A, \text { Base }}$, then $w \notin G_{\text {Base }} / \overline{A P}_{A, \text { Base }}$.

Proof. From the definition, $A P_{A, \text { Base }}=\left\{v \in V(A) \mid G_{\text {Base }} / v \neq G_{A} / v\right\}$, so $\overline{A P}_{A, \text { Base }}=\left\{v \in V(A) \mid G_{B a s e} / v=G_{A} / v\right\}$. Using the Decomposition Lemma, we have:

$$
\begin{aligned}
G_{\text {Base }} / \overline{A P}_{A, \text { Base }} & =G_{\text {Base }} /\left\{v \in V(A) \mid G_{\text {Base }} / v=G_{A} / v\right\} \\
& =\underset{v \in V(A) \mid\left(G_{\text {Base }} / v=G_{A} / v\right)}{\cup} G_{\text {Base }} / v
\end{aligned}
$$

But if for some $v, w \in V\left(G_{\text {Base }} / v\right)$, then $G_{\text {Base }} / w \subseteq G_{\text {Base }} / v$; because $G_{\text {Base }} / w \neq G_{A} / w$, $G_{\text {Base }} / v \neq G_{A} / v$. Hence $w \notin G_{B a s e} / \overline{A P}_{A, \text { Base }}$.

LEMMA (PRESERVED BEHAVIOR LEMMA). Let

$G_{M}=\left(G_{A} / A P_{A, B a s e}\right) \cup\left(G_{B} / A P_{B, \text { Base }}\right) \cup\left(G_{\text {Base }} /\left(\overline{A P}_{A, \text { Base }} \cap \overline{A P}_{B, \text { Base }}\right)\right)$. Then $G_{\text {Base }} /\left(\overline{A P}_{A, \text { Base }} \cap \overline{A P}_{B, \text { Base }}\right)=G_{M} /\left(\overline{A P}_{A, \text { Base }} \cap \overline{A P}_{B, \text { Base }}\right)$.

PROOF. Let $P R E=G_{B a s e} /\left(\overline{A P}_{A, \text { Base }} \cap \overline{A P}_{B, \text { Base }}\right)$ and $P R E^{\prime}=G_{M} /\left(\overline{A P}_{A, \text { Base }} \cap \overline{A P}_{B, \text { Base }}\right)$. Suppose $P R E \neq P R E^{\prime}$. Because $G_{M}$ is created by unioning $P R E$ with $G_{A} / A P_{A, B a s e}$ and $G_{B} / A P_{B, \text { Base }}$, and the slices that generate $P R E$ and $P R E^{\prime}$ are both taken with respect to the same set, $\overline{A P}_{A, B a s e} \cap \overline{A P}_{B, B a s e}$, it must be that $P R E \subset P R E^{\prime}$.

Thus, there are three cases to consider: either $P R E^{\prime}$ contains an additional vertex, an additional control or flow edge (in the latter case either loop independent or loop carried), or an additional def-order edge.

Case 1. $P R E^{\prime}$ contains an additional vertex. Because the slices that generate $P R E$ and $P R E^{\prime}$ are both taken with respect to the set, $\overline{A P}_{A, \text { Base }} \cap \overline{A P}_{B, \text { Base }}, P R E^{\prime}$ can only contain an additional vertex $v$ if there is an additional control or flow edge $v \rightarrow_{c, f} w$ whose target $w$ is an element of both $P R E$ and $P R E^{\prime}$. Thus, this case reduces to the one that follows, in which $P R E^{\prime}$ contains an additional flow edge.

Case 2. $P R E^{\prime}$ contains an additional control or flow edge. The slice operation is a backward closure; because the slices that generate $P R E$ and $P R E^{\prime}$ are both taken with respect to the same set, namely $\overline{A P}_{A, B a s e} \cap \overline{A P}_{B, B a s e}$, if $P R E^{\prime}$ were to contain control or flow edges not in $P R E$, then there is at least one such edge whose target vertex occurs in both $P R E$ and $P R E^{\prime}$. That is, there is at least one edge $e: v \rightarrow w$, where $e \in E\left(P R E^{\prime}\right), v, w \in V\left(P R E^{\prime}\right), w \in V(P R E)$, and $v \notin V(P R E)$.

Because $G_{M}$ is created by a graph union, $e$ must occur in $E\left(G_{A} / A P_{A, B a s e}\right)$, $E\left(G_{B} / A P_{B, B a s e}\right)$, or both. Without loss of generality, assume that $e \in E\left(G_{A} / A P_{A, B a s e}\right)$, so that $e \in E\left(G_{A}\right)$. 
The slice operation is a backward closure, so $e \notin E(P R E)$ and $w \in V(P R E)$ imply $e \notin E\left(G_{B a s e}\right)$. Taking this together with the previous observation that $e \in E\left(G_{A}\right)$, we conclude, from the definition of $A P_{A, B a s e}$, that $w \in V\left(A P_{A, B a s e}\right)$.

This yields a contradiction as follows. By the previous lemma, $w \in V\left(A P_{A, B a s e}\right)$ implies that $w \notin V\left(G_{\text {Base }} / \overline{A P}_{A, \text { Base }}\right)$. However, $G_{\text {Base }} /\left(\overline{A P}_{A, \text { Base }} \cap \overline{A P}_{B, \text { Base }}\right) \subseteq G_{\text {Base }} / \overline{A P}_{A, \text { Base }}$, which means that $w \notin V(P R E)$.

Case 3. $P R E^{\prime}$ contains an additional def-order edge. Suppose $E\left(P R E^{\prime}\right)$ contains a def-order edge $e: v \rightarrow_{d o(u)} w$ that does not occur in $E(P R E)$. By the definition of the edge set of a slice, there must exist flow edges $v \rightarrow_{f} u$ and $w \rightarrow_{f} u$ in $E\left(P R E^{\prime}\right)$; by case (2), these edges must occur in both $E(P R E)$ and $E\left(P R E^{\prime}\right)$ (implying that $u, v, w \in V(P R E), V\left(P R E^{\prime}\right)$ ).

Because $G_{M}$ was created by a graph union, $e$ must occur in $E\left(G_{A} / A P_{A, B a s e}\right)$, $E\left(G_{B} / A P_{B, B a s e}\right)$, or both. Without loss of generality, assume that $e \in E\left(G_{A} / A P_{A, B a s e}\right)$, so that $e \in E\left(G_{A}\right)$.

The slice operation is a backward closure, so $e \notin E(P R E)$ and $u \in V(P R E)$ imply $e \notin E\left(G_{\text {Base }}\right)$; by the definition of $A P_{A, \text { Base }}$, we conclude that $u \in V\left(A P_{A, B a s e}\right)$.

This yields a contradiction analogous to the one that arose in case (2): by the lemma, $u \in V\left(A P_{A, B a s e}\right)$ implies that $u \notin V\left(G_{\text {Base }} / \overline{A P}_{A, \text { Base }}\right)$. However, $G_{\text {Base }} /\left(\overline{A P}_{A, \text { Base }} \cap \overline{A P}_{B, \text { Base }}\right) \subseteq G_{\text {Base }} / \overline{A P}_{A, \text { Base }}$, which means that $u \notin V(P R E)$.

We conclude that $P R E$ and $P R E^{\prime}$ cannot differ; that is, even if variants $A$ and $B$ interfere with respect to base program $B$ ase, the slice $G_{B a s e} /\left(\overline{A P}_{A, \text { Base }} \cap \overline{A P}_{B, \text { Base }}\right)$ is preserved in $G_{M}$.

The base program, the two variants, and the merged program share common slices; thus, the next matter to address is the relationship between the execution behaviors of two programs when they share a common slice. An immediate consequence of the Slicing Theorem is that two programs that share a slice agree on all variables for which there are final-use vertices in the slice.

SLICING COROLLARY. Let $P$ and $Q$ be two programs that share a slice with respect to a set of program points $S$ (i.e. $P / S$ is isomorphic to $Q / S$ ). Then, for any initial state $\sigma$ on which both $P$ and $Q$ halt, the final states produced by $P$ and $Q$ agree on all variables for which there are final-use vertices in $S$.

Using the Slicing Corollary, the definition of the merged graph $G_{M}$, and the Preserved Behavior Lemma, we can now characterize the execution behavior of the integrated program in terms of the behaviors of the base program and the two variants.

THEOREM. (INTEGRATION THEOREM). If $A$ and $B$ are two variants of Base for which integration succeeds (and produces program $M$ ), then for any initial state $\sigma$ on which $A, B$, and Base all halt, (1) $M$ halts on $\sigma,(2)$ if $x$ is a variable defined in the final state of $A$ for which the final states of $A$ and Base disagree, then the final state of $M$ agrees with the final state of $A$ on 
$x$, (3) if $y$ is a variable defined in the final state of $B$ for which the final states of $B$ and Base disagree, then the final state of $M$ agrees with the final state of $B$ on $y$, and (4) if $z$ is a variable on which the final states of $A, B$, and Base agree, then the final state of $M$ agrees with the final state of Base on $z$.

Proof. We use $A / A P_{A, B a s e}, B / A P_{B, \text { Base }}$, and Base $/\left(\overline{A P}_{A, \text { Base }} \cap \overline{A P}_{B, \text { Base }}\right)$ to denote programs whose program dependence graphs are $G_{A} / A P_{A, B a s e}, G_{B} / A P_{B, \text { Base }}$, and $G_{\text {Base }} /\left(\overline{A P}_{A, \text { Base }} \cap \overline{A P}_{B, \text { Base }}\right)$, respectively.

Since the integration succeeds, $G_{A} / A P_{A, B a s e}=G_{M} / A P_{A, \text { Base }}$ and $G_{B} / A P_{B, \text { Base }}=$ $G_{M} / A P_{B}$, Base. $B y$ the Preserved Behavior Lemma, $G_{\text {Base }} /\left(\overline{A P}_{A, B a s e} \cap \overline{A P}_{B, \text { Base }}\right)=$ $G_{M} /\left(\overline{A P}_{A, \text { Base }} \cap \overline{A P}_{B, \text { Base }}\right)$. Therefore, we have

$G_{M}=G_{A} / A P_{A, B a s e} \cup G_{B} / A P_{B, \text { Base }} \cup G_{\text {Base }} /\left(\overline{A P}_{A, \text { Base }} \cap \underline{A P}_{B, \text { Base }}\right)$

$=G_{M} / A P_{A, \text { Base }} \cup G_{M} / A P_{B, \text { Base }} \cup G_{M} /\left(\overline{A P}_{A, \text { Base }} \cap \overline{A P}_{B, \text { Base }}\right)$.

Since $A$ halts on $\sigma$, by the Slicing Theorem $A / A P_{A, B a s e}$ also halts on $\sigma$. Similarly, $B / A P_{B, \text { Base }}$ and Base $/\left(\overline{A P}_{A, B a s e} \cap \overline{A P}_{B}\right.$, Base $)$ halt on $\sigma$, as well. Note that $A / A P_{A}$,Base $B / A P_{B, \text { Base }}$, and Base $/\left(\overline{A P}_{A, B a s e} \cap \overline{A P}_{B, \text { Base }}\right)$ are programs whose program dependence graphs are $G_{M} / A P_{A, B a s e}, G_{M} / A P_{B, \text { Base }}$, and $G_{M} /\left(\overline{A P}_{A, \text { Base }} \cap \overline{A P}_{B, \text { Base }}\right)$, respectively. Since $A / A P_{A, \text { Base }}, B / A P_{B, B a s e}$, and Base $/\left(\overline{A P}_{A, B a s e} \cap \overline{A P}_{B, \text { Base }}\right)$ halt on $\sigma$, by the Corollary of the Termination Theorem, $M$ halts on $\sigma$. (This demonstrates clause (1) of the Integration Theorem.)

Let $x$ be a variable defined in the final state of $A$ on which the final states of $A$ and $B a s e$ disagree. Let $v$ be the final-use vertex of $x$, so $v \in V\left(G_{A}\right)$. By the Slicing Theorem, $G_{B a s e} / v \neq G_{A} / v$. Therefore, $v \in A P_{A, B a s e}$. Since $v \in A P_{A, B a s e}$ and $G_{A} / A P_{A, B a s e}=$ $G_{M} / A P_{A, B a s e}$, by the Slicing Corollary the final state of $M$ agrees with the final state of $A$ on $x$. (This demonstrates clause (2) of the Integration Theorem.) Similarly, if $y$ is a variable defined in the final state of $B$ on which the final states of $B$ and $B a$ se disagree, then the final state of $M$ agrees with the final state of $B$ on $y$. (This demonstrates clause (3) of the Integration Theorem.)

What remains to be shown is that if $z$ is a variable on which the final states of $A, B$, and $B$ ase agree, then the final state of $M$ agrees with the final state of Base on $z$. Let $v$ be the final-use vertex of $z$. If $v \in A P_{A, \text { Base }}$, since $G_{A} / A P_{A, B a s e}=G_{M} / A P_{A, B a s e}$, by the Slicing Corollary, the final state of $M$ agrees with the final state of $A$ on $z$, which means the final state of $M$ agrees with the final state of Base on $z$. Similarly, if $v \in A P_{B, B a s e}$, since $G_{B} / A P_{B, \text { Base }}=$ $G_{M} / A P_{B, B a s e}$, by the Slicing Corollary, the final state of $M$ agrees with the final state of $B$ on $x$, which means the final state of $M$ agrees with the final state of Base on $x$. Finally, since $v$ is a final-use vertex of $G_{M}$, if $v \notin A P_{A, B a s e}$ and $v \notin A P_{B, \text { Base }}$, then $v \in \overline{A P}_{A, \text { Base }} \cap \overline{A P}_{B, B a s e}$. Because $G_{\text {Base }} /\left(\overline{A P}_{A, \text { Base }} \cap \overline{A P}_{B, \text { Base }}\right)=G_{M} /\left(\overline{A P}_{A, \text { Base }} \cap \overline{A P}_{B, \text { Base }}\right)$, by the Slicing Corollary the final state of $M$ agrees with the final state of Base on $x$. (This demonstrates clause (4) of the Integration Theorem.) 


\section{RELATION TO PREVIOUS WORK}

This paper continues the study of program dependence graphs and program semantics begun in [3]. The Equivalence Theorem proven in [3] addresses the relationship between isomorphic PDGs; the Equivalence Theorem shows that if the program dependence graphs of two programs are isomorphic then the programs are strongly equivalent (i.e. given the same initial state, either both diverge or both halt in the same final state). By contrast, the Slicing and Termination Theorems proven in this paper concern non-isomorphic PDGs.

Earlier work on program slicing includes [10], [11], and [5]. All of this previous work imposes the condition that a slice be a program whose statements are in the same relative order that they are in the original program. The notion of a slice presented in this paper is a more liberal one: the slice of a program $P$ with respect to a set of program points $S$ is any program $Q$ whose PDG is isomorphic to $G_{P} / S$. In particular, the relative order of $Q$ 's statements is not necessarily the same as in $P$. This generalization is justified by the Equivalence Theorem from [3] together with the Feasibility Lemma from this paper.

A version of the Slicing Theorem (for the more limited notion of slice described above) was demonstrated in [10]; it applies to a different algorithm for extracting slices, based on solving a sequence of data-flow problems, rather than the one studied here, which is based on walking backwards over the edges of a program dependence graph.

The idea of extracting a program slice by walking backwards over dependence edges appears in [5], although that paper gives no justification for the operation. The Feasibility Lemma proven in this paper, (which demonstrates that any slice of a feasible program dependence graph is itself a feasible graph), together with the Slicing Theorem provide the necessary syntactic and semantic justifications, respectively, for this method of extracting slices.

When def-order dependences are used in program dependence graphs, larger classes of strongly equivalent programs have isomorphic program dependence graphs than when output dependences are used [3]. Thus, our use of def-order dependences in place of the more usual output dependences increases the number of programs that are an acceptable outcome for a given slicing operation. (For instance, the following programs are examples of two strongly equivalent programs whose PDGs are isomorphic if def-order dependences are used, but are not isomorphic if output dependences are used:

\begin{tabular}{|c|c|}
\hline $\begin{aligned} & \text { program } \\
& x:=0 \\
& a:=x \\
& x:=1 \\
& b:=x \\
& x:=2 \\
& \text { end }(a, b, x)\end{aligned}$ & $\begin{aligned} & \text { program } \\
& x:=1 \\
& b:=x \\
& x:=0 \\
& a:=x \\
& x:=2 \\
& \text { end }(a, b, x)\end{aligned}$ \\
\hline
\end{tabular}

The program dependence graphs for these programs have the same (empty) set of def-order dependences, but have different sets of output dependences.) 
Finally, this paper provides semantic justification for the program-integration algorithm presented in [2,7]; the Integration Theorem demonstrates that the program that results from a successful integration operation preserves the changed behaviors of the two variants as well as the unchanged behavior of the base program.

\section{REFERENCES}

1. Ferrante, J., Ottenstein, K., and Warren, J., "The program dependence graph and its use in optimization," ACM Transactions on Programming Languages and Systems 9(3) pp. 319-349 (July 1987).

2. Horwitz, S., Prins, J., and Reps, T., "Integrating non-interfering versions of programs," pp. 133-145 in Conference Record of the Fifteenth ACM Symposium on Principles of Programming Languages, (San Diego, CA, January 13-15, 1988), ACM, New York, NY (1988).

3. Horwitz, S., Prins, J., and Reps, T., "On the adequacy of program dependence graphs for representing programs," pp. 146-157 in Conference Record of the Fifteenth ACM Symposium on Principles of Programming Languages, (San Diego, CA, January 13-15, 1988), ACM, New York, NY (1988).

4. Kuck, D.J., Muraoka, Y., and Chen, S.C., "On the number of operations simultaneously executable in FORTRAN-like programs and their resulting speed-up," IEEE Trans. on Computers C-21(12) pp. 1293-1310 (December 1972).

5. Ottenstein, K.J. and Ottenstein, L.M., "The program dependence graph in a software development environment," Proceedings of the ACM SIGSOFT/SIGPLAN Software Engineering Symposium on Practical Software Development Environments, (Pittsburgh, PA, Apr. 23-25, 1984), ACM SIGPLAN Notices 19(5) pp. 177-184 (May 1984).

6. Reps, T. and Teitelbaum, T., "The Synthesizer Generator," Proceedings of the ACM SIGSOFT/SIGPLAN Software Engineering Symposium on Practical Software Development Environments, (Pittsburgh, PA, Apr. 23-25, 1984), ACM SIGPLAN Notices 19(5) pp. 42-48 (May 1984).

7. Reps, T. and Horwitz, S., "Semantics-based program integration," pp. 1-20 in Proceedings of the Second European Symposium on Programming, (Nancy, France, March 21-24, 1988), Lecture Notes in Computer Science, Vol. 300, ed. H. Ganzinger,Springer-Verlag, New York, NY (1988).

8. Reps, T. and Teitelbaum, T., The Synthesizer Generator: A System for Constructing Language-Based Editors, Springer-Verlag, New York, NY (1988).

9. Reps, T. and Yang, W., "The semantics of program slicing," TR-777, Computer Sciences Department, University of Wisconsin, Madison, WI (June 1988).

10. Weiser, M., "Program slices: Formal, psychological, and practical investigations of an automatic program abstraction method.," Ph.D. dissertation, Univ. of Michigan, Ann Arbor, MI (1979). As reported by personal communication from M. Weiser, July 1988.

11. Weiser, M., "Program slicing," IEEE Transactions on Software Engineering SE-10(4) pp. 352-357 (July 1984). 